\title{
Reconsidering the encoding of data in physics education research
}

\author{
R. Padraic Springuel ${ }^{*}$ \\ St. Anselm's Abbey, 4501 South Dakota Avenue Northeast, Washington, DC 20017, USA \\ Michael C. Wittmann and John R. Thompson \\ Department of Physics and Astronomy and Maine Center for Research in STEM Education, \\ University of Maine, Orono, Maine 04469, USA
}

(Received 31 August 2018; published 3 July 2019)

\begin{abstract}
[This paper is part of the Focused Collection on Quantitative Methods in PER: A Critical Examination.] How data are collected and how they are analyzed is typically described in the literature, but how the data are encoded is often not described in detail. In this paper, we discuss how data typically gathered in PER are encoded and how the choice of encoding plays a role in data analysis. We describe the kinds of data that are found when using short answer, multiple choice, Likert-scale, ranking task, and free response questions in terms of nominal, ordinal, interval, and ratio data. We discuss the mathematical operations that are available for each kind of data and how this affects ways that similarity and difference between student responses can be determined, a topic we discuss in terms of measures of distances and correlation. Finally, we use several papers from the literature to discuss ways in which data have been encoded and analyzed, with examples of normalized gain, factor analysis, model analysis, cluster analysis, and the investigation of epistemological agreement. We highlight both strengths and weaknesses of the data encoding approaches used in these studies. Our goal is not a comprehensive review, but one that is illustrative and can help researchers understand their own and each other's work more deeply.
\end{abstract}

DOI: 10.1103/PhysRevPhysEducRes.15.020103

\section{INTRODUCTION}

Many kinds of research in physics education research (PER) involve connecting a research question to observations of student behavior or knowledge in such a way that the information contained in the observations can be used to answer the research question. This connection can be thought of as building a chain between these two end points. Along the way there are several decisions, or links, which must be made and the way we make those decisions affects the strength of the links we are forging. Several of these links are quite obvious and well discussed in the existing literature. For instance, tests like the Force and Motion Conceptual Evaluation (FMCE) and Force Concept Inventory (FCI) have been designed as tools with which certain patterns of student behavior or knowledge can be measured in some populations. This would be an example of the first link in the chain on the student behavior or knowledge side, data gathering, by which we restrict ourselves from making all possible observations, to just

\footnotetext{
*rpspringuel@gmail.com
}

Published by the American Physical Society under the terms of the Creative Commons Attribution 4.0 International license. Further distribution of this work must maintain attribution to the author(s) and the published article's title, journal citation, and DOI. the one which we believe is relevant to answering our research question. In order to be strong, this link must both measure what it claims to measure (be valid) and give repeatable results (be reliable). On the other end of the chain, we might consider a research question that asks about the relative effectiveness of two different curricula in promoting student learning. The first link on this side might be to select a comparison of normalized gains, a form of data analysis. The strength of this link would be determined by things like statistical power and effect size.

Of course, these links are not forged in isolation. The data gathering link must be directed towards collecting data that is relevant to the research question; they must be connectable. To use another analogy, if we are trying to build a bridge from France to England across the Channel, then it makes no sense to lay the French foundations in Nice (along the Mediterranean). That is, given the research question, a researcher will try to pick the data which is most likely to provide an answer to that question. To do otherwise would be absurd. On occasion, other researchers might question whether some other data set might not be more suitable and thus start a discussion about degree of connectedness between the data and the research question (as mediated through the analysis method), but the focus of this discussion tends to be on the inherent connectability of the data to the analysis method and through that to the research question. Whether or not this suitability discussion 
takes place, however, there is a step which is often overlooked: once it has been established that the data can be connected to the analysis method and research question, it then remains to establish how to make that connection as strong as possible. It is the goal of this paper to discuss what goes into the establishment of a strong connection between the data and the analysis method: to examine the data encoding process in and of itself.

The "data" typically presented in PER papers and presentations are not actually data but rather an encoding of data [1]. In qualitative research, this seems obvious; text-only transcripts of interviews do not contain all the information from the interview (such as gesture, intonation of speech, tone of voice, or stress on particular words), and even strongly annotated transcripts do not contain all the information. The transcript is a kind of encoding of the salient information, as chosen by the researcher, which helps convey the desired information while leaving out information that is deemed unimportant for the argument being made. In quantitative research, data are similarly encoded. As the purpose of this special collection is the examination of quantitative data, this paper will look at the specifics of encoding such data, how it affects the information that is either kept or lost from the original data, and how the decisions about encoding affect the arguments and claims that can be made based on the data.

In presenting the results of quantitative research, data source selection, data collection, and data analysis are usually discussed in detail. Further, there is usually some discussion of how these three steps go together and how they serve the research question. However, data encoding is often neglected or taken for granted, even though this step forms an important bridge between the data collection and analysis. It is important that one fairly represents the data so that the chosen encoding maps reasonably well onto the information collected. It is also important that the properties of the encoded data line up with the assumptions made as part of data analysis. Without both of these sources of consistency, the results of our research can be limited in their interpretation and generalizability. While there may be some circumstances in which the limitations created by some forms of inconsistency between the data collection, data encoding, and data analysis do not hinder a researcher's ability to answer the research question at hand, we do not intend to treat those circumstances here. Such circumstances are, by their nature, specific to the research question and thus present far too many possibilities for us to account for in this paper.

This paper describes both the abstract theory of data and concrete examples from PER to discuss the process of moving from gathering data to encoding it to analyzing it. We begin by describing the properties of different forms of data and how these relate to the encoding of data. We describe how these properties correspond to the information being represented, and how these properties affect the sorts of mathematical operations that are permissible on the data. For some forms of data, certain mathematical operations are not possible, simply due to the nature of the data. One cannot take the average of the responses to a multiple choice question, for example; the mode of these responses would be more appropriate.

We follow this discussion with an example-driven, practical discussion of how to maintain consistency between the data collection and data encoding steps of quantitative research. We consider some of the basic types of questions that have been asked on written surveys in PER (e.g., multiple-choice, free response, Likert-scale, ranking task) and describe what sort of information each yields. We map this back on to what we would consider to be the optimal type of encoding to use with that question.

We then use an example-driven, practical approach to look at how to maintain consistency between the data encoding and data analysis steps. We focus particularly on distance measures and correlation coefficients in our discussion as these form the fundamental first step for several different analysis methods. We assess how these distances or correlations treat their data and thus what properties they (and any analysis method based on them) are assuming the data to have. This will allow us to indicate what we believe to be the optimal type of data encoding to use with that distance or correlation.

Finally, we review some of the most prominent methods of quantitative data analysis using a select group of papers from the PER literature to discuss the process of encoding and analysis in detail. We look at examples of using normalized gain, factor analysis, model analysis, cluster analysis, and the study of epistemological agreement. This allows us to highlight cases where there is close alignment between the data, its encoding, and the analysis, as well as cases where information is lost in the encoding, and its consequences on subsequent analysis.

Given the amount of published work in PER, our work is not comprehensive. Even within the restrictions of written surveys analyzed with a method which is based on a distance measure or correlation coefficient, there are far too many possible research questions for us to address in a paper, like this one. Instead, with this review paper, and its necessarily limited discussion of data encoding in a small slice of the existing PER literature, we seek to initiate a discussion of encoding processes in order to stimulate the PER community to take this procedural step more seriously and feature it more prominently in publications and presentations.

\section{THEORY OF DATA}

Before we can consider the particular issues which present themselves in encoding specifically PER data, we need a common theory of data which establishes the terminology that we are going to use, what those terms mean, and how they relate to each other. The below 
discussion of a theory of data is necessarily an overview of the topic geared especially for PER considerations. For a more in depth discussion of a theory of data, the reader should refer to textbooks on statistics and data analysis. In the discussion below we have cited those we have made use of and considered to be helpful.

Different kinds of questions are not created equal in terms of the kind and quality of student knowledge that they probe; so too, different kinds of data do not contain the same amount of information. Stated differently, the number of different comparisons that can be made between two different instances of the datum varies. The more comparisons that can be made, the more information that datum is said to contain. It is, of course, often trivially easy to see that two responses are not identical. It is, however, considerably more complicated to determine which of the two is more similar (or different) from a third nonidentical response. However, just such a consideration is the basis for many types of quantitative analysis (e.g., cluster analysis, factor analysis, classification, and model analysis). The task of determining just how similar (or different) responses are from each other (and in comparison to a third point) is what we are most interested in answering when dealing with quantitative data. We need, therefore, to start by looking at the properties, particularly the mathematical properties, of the data which can form the basis for devising an answer to that question.

\section{A. Mathematical properties of data}

In order to determine the amount and quality of information in a particular kind of data, there are three mathematical properties that should be examined: the number of states that the data can take on (also referred to as data type or range set) [2-6], the scale on which those states are measured [2-5], and the symmetry (or uniformity) of the first two properties [3,4].

The examination of the number of states (or values) that a piece of data can take on is a determination of the size of the data's range set. As such, there are three mathematically distinct classes of range set that appear: finite (a set with a limited number of elements), countably infinite (a set with an unlimited number of elements but where the number of elements between any two elements is limited), and uncountably infinite (a set where both the number of elements in total and the number of elements in between any two elements is unlimited) [2]. Data analysis, however, has not traditionally started from that mathematical basis. Instead it classifies numbers of values into three types: binary (datum can take on one of two values), discrete (datum can only take on specific allowed values), and continuous (datum can take on any value, sometimes within a limited range) [2-6]. This classification scheme, however, has several problems. First, discrete conflates sets that are finite and countably infinite $[2,4]$ with the results that in some cases one or the other is overlooked. For example,
Dubes and Jain [6] (and Jain and Dubes [5]) describes discrete as only having a finite number of values. Second, binary is a special case of finite in which only two values (as opposed to an arbitrary number) are allowed [4]. There is no theoretical, mathematical reason why data with two states should be treated differently from those with three or more [7]. Third, the precision limits of any instrument mean that while a particular datum might have an uncountably infinite range set in theory, in practice it has a countably infinite one. Its uncountable nature only manifests itself in the possibility of developing a more precise instrument, not in the nature of the data collected. As a result, it makes more theoretical sense to think of the number of states as falling into two types: finite (there is a limited, enumerable set of states) and infinite (data can take on any value).

The measurement scale of a datum determines what relations it has with other data [5], in other words, which mathematical operations are well defined on the data set [4]. These mathematical operations include distinctness $(=$ and $\neq)$, order $(<$ and $>)$, addition $(+$ and -$)$, and multiplication $(\times$ and $\div$ ) $[4,8]$. In each instance, these operations are cumulative. If a higher-order operation is well defined, then all lower-order operations are also well defined (e.g., if we can tell relative order between two data, then we can also determine whether they are distinct from each other). As a result, four different measurement scales have been identified: nominal (only distinctness is well defined), ordinal (both order and distinctness are well defined), interval (distinctness, order, and addition are well defined), and ratio (distinctness, order, addition, and multiplication are well defined). These four scales are thus generally considered hierarchical with nominal at the bottom (containing the least amount of information) and ratio at the top (containing the most amount of information) [2,4,5] [11].

Symmetry is not really a separate property of data, but rather a modification on either the property of number or scale. In some cases a data set may have certain states that do not have the same properties as other states. Such states are considered to be asymmetric and lead to special modifications to data handling processes in order to account for them. While any conceivable algorithm that leads to different states being treated as not equivalent is a form of asymmetry, there are two forms of asymmetry that are of particular interest in PER. By far the most common type of asymmetric state is the case of missing data. When a datum is missing, comparisons to other data, be they missing or not, are impossible. As a result, some process must be devised that handles the missing data in a special (and necessarily different) way [3,5]. A second common type of asymmetric state, which occurs frequently in PER, is the "opt-out" response. In questions that provide a set of responses (such as multiple-choice questions), students may be given the option to indicate that their desired response is not represented in the available responses 
("None of the above") [14]. This is different from missing data because the response indicates disagreement with all other available states. At the same time, however, it is similar to missing data because it is impossible to compare this state across students (two students who both chose none of the above may do so for dramatically different reasons). As a result, distinctness $(=$ and $\neq$ ) is only partially defined. This must be accounted for in data analysis.

\section{B. Types of data}

While in principle it is possible to consider number, scale, and symmetry independently of each other and thus come up with a huge number of kinds of data (infinite when we consider the countless ways of creating asymmetries), in practice things turn out to be far simpler, especially in PER. First off, a given data set is necessarily finite; thus the difference between finite range set and infinite range set is more a question about how new data are added to the data set. If the range set is finite, then the researcher should be able to enumerate all the possible data states before any encoding is done and thus new data will fall into one of the existing known states. On the other hand, if the range set is infinite, a complete enumeration of the data states is not possible. Any new data necessarily contain the possibility of having new, previously unknown, data states. As a result, one has to develop rules for determining what qualifies as a new data state as opposed to a new instance of an existing state. In both cases we are dealing with process considerations: what are the steps taken to ensure a consistent encoding of the data. Once the data are encoded, however, the finite nature of the data set means that the encoded data will have the same properties, scale and symmetry being equal. Furthermore, except for the two cases described above (missing data and the asymmetric nominal option), data are usually treated symmetrically. As a result, this paper will treat missing data in Sec. II C as a separate concern from the type of data and will define five different types of data based primarily on measurement scale: asymmetric nominal, symmetric nominal, ordinal, interval, and ratio.

\section{Asymmetric nominal}

Consider an experiment in which it is necessary to categorize light striking a photodetector based on its color. One might have the categories "red," "orange," "yellow," "green," "blue," "indigo," "violet," and "invisible." Each of the first seven categories reveals something about the color of the light, providing a rough idea of the energy of the photons that the photodetector is counting. However, invisible reveals no such thing. The photons could be high energy (ultraviolet and above) or low energy (infrared or below). The only thing "known" is that there are photons striking the photodetector (i.e., the measurement opportunity was not missed), not how to classify that light.
These are asymmetric nominal data. Such data are defined by two characteristics: a set of finite, distinct possible responses and the presence within that set of a single option which provides "uneventful" information [3] (p. 27). For this reason asymmetric nominal data are considered to contain the least amount of information: the information provided is not always useful and is confined to being in one of the predefined categories. Mathematically this means that distinctness $(=$ and $\neq)$ can be established for asymmetric nominal data, but not all the time (i.e., when comparing the state with uneventful information to itself). Typical examples from (PER) are of multiple-choice questions containing the option none of the above, where two students may select said option but be looking for completely different responses.

\section{Symmetric nominal}

Consider, in contrast, descriptions of the motion of a moving object. It might be "speeding up," "slowing down," or "moving at constant speed." Each category provides information about an object's acceleration sign relative to that of its velocity. The set is complete (there are no other options), but there are still a limited number of categories: 3 .

Such symmetric nominal data are similar to asymmetric nominal data in that there is a finite, distinct set of possible responses, but unlike asymmetric data, all of the categories provide "equally important" information [3]. As a result, distinctness $(=$ and $\neq)$ can always be established for symmetric nominal data. Because each category contains useful information, symmetric nominal data in general contain more information than asymmetric nominal data. A specific asymmetric nominal data point might have more information than a specific symmetric nominal one by virtue of having more categories available for classification, but, given the same number of categories, on average there will be more information stored in each symmetric nominal data point.

\section{Ordinal}

As with both types of nominal data, ordinal data contain a finite, distinct set of possible responses. However, unlike nominal data, these responses have order relations with each other [3]. This means that in addition to distinctness there is also a concept of order $(<$ and $>)$. The order in which a particular student does the problems on a test is an example of ordinal data (in which case "greater than" and "less than" would map to "after" and "before"). This order can come in two forms: within students and between students.

Within-students ordinal data arises when each student is asked to rank some set of items according to some external criteria. In these instances, a comparison of one student to another should take into account the whole ranking scheme and not just be an individual item-by-item comparison. 
This is necessary because any displacement in the list that is not a swap (two items switching ranks) will affect the rank of every other item on the list after it. An item-by-item comparison would make this change in the ranking seem more extreme than warranted. For example, the rank orders of $A<B<C<D<E$ and $A<C<D<E<B$ only differ in their placement of $B$ and yet an item-by-item comparison says four of the five items have different ranks in the two schemes. On the other hand, $A<E<D<C<B$ also has four of five items with different ranks, but has changed many more order relations and thus is more different. By examining the whole ranking scheme at once we can account for these kinds of differences.

Between-students ordinal data occur when some external criteria ranks students against each other, such as when recording the order of finish to a test. While such data are ordinal, each student has only one rank, making it impossible to compare students by examining whole ranking schemes. However, the difference between two students' ranks does have a meaningful interpretation: it is one more than the number of ranks (other students) that came between them [15]. This meaning can be exploited to treat between-students ordinal data as interval data (described below).

Not all sets of ordinal data are perfectly ordered. In some cases it is possible for multiple items to be indistinguishable from each other according to the criteria used to rank them (i.e., they are tied at the same rank). In dealing with ties in within-students ordinal data, it is conventional to give to the tied items the average of the ranks that would be awarded were they distinguishable (i.e., a two-way tie for rank 2 would result in two ranks of 2.5 , the average of ranks 2 and 3). In this way, (i) the indistinguishable items are given indistinguishable ranks and (ii) the sum of the ranks for all members is the same as it would be if the items were distinguishable $(1+2+3+4=10=1+2.5+2.5+4)$ [16]. Known as the "mid-rank method," this convention is different from how ties are dealt with in other ordered lists in everyday life (e.g., a list of favorite authors). In the everyday convention, the tied data points are all given the best rank (usually the lowest) and then a number of ranks equal to one less than the number of tied items are skipped (e.g., I might have two authors whom I like equally well in my number 2 spot, in which case I have two second favorite authors, no third favorite, and then continue the list with a fourth favorite). This follows the first criteria, but fails the second $(1+2+3+4=10 \neq 9=1+2+2+4)$. On the other hand, when dealing with between-students rankings, the mid-rank method is actually counterproductive because it does not respect the interpretation of the rank difference described above. Instead, in keeping with this meaning for the rank difference, each of the tied students should be given the rank that would have been assigned if there had only been one student in that position and the rank assigned to the following students should not account for the presence of multiple students at the same rank (i.e., the two-way tie for rank 2 would result in a rank order of 1,2 , $2,3$, not $1,2,2,4$, or $1,2.5,2.5,4)$ [17].

\section{Interval}

Temperatures measured in Celsius or Fahrenheit, calendar dates, or times of the day all are common examples of interval data. Interval data take ordinal data one step further by valuing the difference between two places in the order [4]. This means that distinctness, order, and addition $(+$ and -$)$ are well defined and there are an infinite number of states (though only a finite subset is used in any given data set).

\section{Ratio}

While it is possible to take the difference between two temperatures when they are measured in degrees Celsius or Fahrenheit, it is not appropriate to take the ratio of two temperatures unless those temperatures are measured on an absolute scale (e.g., Kelvin). With ratio data, the 0 point of the scale represents a complete absence of the property being measured, unlike interval data for which the 0 point may be set arbitrarily. Because multiplication and division are well-defined mathematical operations for ratio data [3], one of the distinctions between 2 and 20 is the same as between 20 and 200 (specifically a scaling factor of 10). Plots of normalized gain on tests like the FCI create a similar comparison of ratios. As with interval data, ratio data have an infinite number of states (though only a finite subset is used in any given data set).

\section{Missing data}

Researchers are frequently constrained to only asking a limited number of questions, and students do not always answer all the questions asked of them. These two problems, which create "holes" in a data set, can be dealt with in a variety of ways. Tan et al. [4] offer several possible solutions, summarized below.

When the holes in a data set are confined to a few students, the simplest way of dealing with these holes is to simply eliminate the affected students from the data set prior to analysis. The information about those students is lost, but the resulting smaller data set will be "complete" in the sense that there are no missing values, and analysis methods will not need special instructions for dealing with the holes [see Fig. 1(a)].

Likewise, if the holes are confined to just a few questions (e.g., a series of questions at the end of an instrument or certain questions that turned out to be extraordinarily difficult), then elimination of the offending questions from our data set before analysis is reasonable. Again, this results in a loss of information, but a more complete data set [see Fig. 1(b)].

Of course, most times the holes will be evenly distributed enough that employing either or both of the above two 


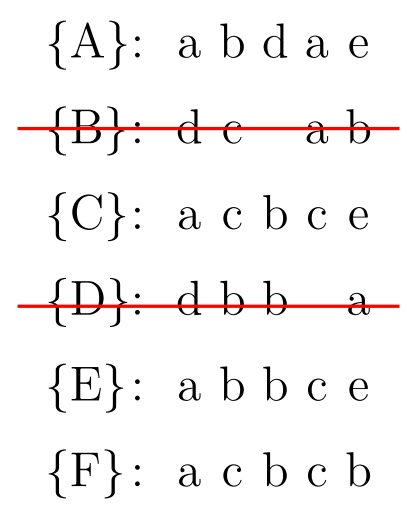

(a)Deleting Students

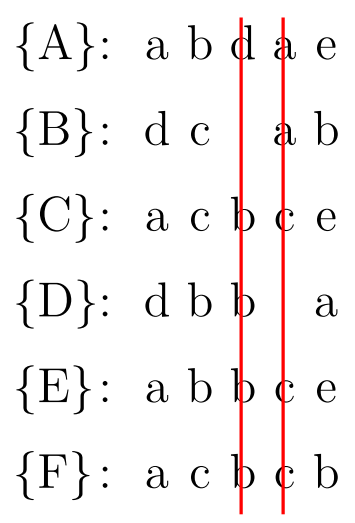

(b)Deleting Questions
$\{A\}:$ a b d a e
$\{B\}:$ d c b a b
$\{C\}:$ a c b c e
$\{D\}:$ d b b c a
$\{E\}:$ a b b c e
$\{F\}:$ a c b c b

(c)Fill-in-the-Blanks

FIG. 1. Strategies for dealing with missing data.

strategies would result in far too much data being lost [18]. When this is the case, there are two other strategies that can come into play (alongside or to the exclusion of the above two strategies and each other).

The first strategy is to fill in the missing data somehow. Perhaps the student's answer to another question (or questions) can indicate what their missing answer would have been. Or it may be that the relevant question has an answer that students can be considered to have given by default, e.g., none of the above. Care must be used when employing this method, however, as any assumptions about how the students would have hypothetically answered a question may create or accentuate similarities or differences that are not actually present in the data [see Fig. 1(c)].

The final strategy is to ignore missing data when necessary. For example, in the data set used in Fig. 1, student $\mathrm{B}$ did not answer question 3 and student $\mathrm{D}$ did not answer question 4 . Therefore in an analysis step which involves both students B and D, neither question 3 nor 4 would be used, effectively reducing the number of questions to 3. At the same time though, during an analysis step which involved only one of students B or D then only question 3 or question 4 would be ignored, as needed. Analysis steps which involve neither students B or D would not ignore any questions. It should be pointed out that those analysis steps which selectively ignore some of the data are only approximations of what they would be were it not for the missing data. However, if the number of missing data points is small relative to the size of the data set, the issue is minimal. Furthermore, the use of fuzzy math (a branch of mathematics in which logical relations are probabilistic rather than deterministic) can explicitly account for the uncertainty introduced by missing data in some cases.

\section{MATCHING DATA TYPES}

Having outlined a basic theory of data, we now connect this theory more explicitly to PER by discussing how the type of data forms the bridge between the student responses gathered using whatever instrument is appropriate to the study being performed and the analysis methods used to extract some actionable conclusions. Ideally, the data type needs to match at both ends. On the one hand, it needs to accurately and concisely represent the range of response which students give to the questions that we ask. A data type that is incapable of representing some feature of the responses collected will hide any significance (or lack thereof) that feature might have, while a data type that stores more information than is present in the responses can potentially introduce effects not actually supported by the responses. On the other hand, different methods of analysis use different mathematical tools; each tool assumes that the data type being fed into that tool has the appropriate properties to make the results of the tool meaningful. Errors in this step, such as using mathematical tools meant for ratio data while only having interval data, are easy to make. In the ideal world, then, the method of analysis would require a data type that accurately and concisely maps onto the responses given by the students. Any deviations should be explicitly acknowledged and justified based on the research question being examined.

While we cannot hope to be exhaustive in the discussion below, we will examine the two ends of this matching process in some detail. First we will look at the process of matching question types to data types. That is, we will consider what kinds of questions are asked in PER on typical written surveys and examine the set of expected responses to figure out what data type can be used to most accurately and concisely represent those responses. Second we will look at the process of quantifying the difference or similarity between two encoded sets of responses and how various ways of doing that demand different types of data. There are, of course, other forms of quantitative analysis that may not depend on how similar or different two sets of responses are. However, this is the basis for many methods of analysis (as we pointed out above), and we hope the 
TABLE I. The natural line up between data types and question types.

\begin{tabular}{lccccc}
\hline \hline Question type & Asymmetric nominal & Symmetric nominal & Ordinal & Interval & Ratio \\
\hline Multiple choice & & & & & \\
$\quad$ Single response & $\mathrm{X}$ & $\mathrm{X}$ & & & \\
$\quad$ Multiple response & $\mathrm{X}$ & $\mathrm{X}$ & & $\mathrm{X}$ & $\mathrm{X}$ \\
Short answer & $\mathrm{X}$ & & $\mathrm{X}$ & & \\
Ranking tasks & & $\mathrm{X}$ & & \\
Likert scale & $\mathrm{X}$ & $\mathrm{X}$ & $\mathrm{X}$ & $\mathrm{X}$ & $\mathrm{X}$ \\
Free response & &
\end{tabular}

more concrete discussion will provide a suitable model for those using other analysis methods to follow (even if the details of what they are doing are different).

\section{A. Matching to questions}

Translating from question types to data types requires encoding the student answers to these questions in a way that preserves as much of the information contained therein as is reasonable. The more information encoded, the more types of analysis are possible and the more nuanced the results of that analysis can be. Moreover, while it is always possible to apply a filter to an encoding to remove information that a particular form of analysis deems irrelevant or is incapable of using, it is not possible to do the reverse. Once an encoding selects a portion of the data to be recorded, only information contained in that portion of the data can be analyzed without re-encoding the data. However, there does come a point when so much information is encoded that it interferes with the researcher's ability to manage the encoding process (e.g., encoding whether a student's handwriting was in cursive script or block lettering). All forms of encoding will exclude some of the features present in the responses as the only way to encode all the features is with an exact duplicate of the original responses. Our goal in encoding the data, therefore, is to exclude those features which are most likely meaningless and include only those features which we expect to be meaningful. As a result it pays to look at each type of question used in PER and what sort of responses we expect to get out of them in order to determine how researchers might reasonably encode student responses to each.

Redish [19] described 9 exam and homework question types: multiple choice, short answer, multiple-choice multiple response (MCMR) (also known as multiple true or false), representation translation, ranking task, context-based reasoning, estimation, qualitative, and essay. However, some of the distinctions between question types are based on the form of the student answer (e.g., multiple choice vs essay), while others are based on the kind of thinking the student must engage in to answer the question (e.g., qualitative vs essay). While the former distinction is appropriate within the data driven view that this paper takes, the latter is not. As a result, we classify the kinds of questions asked in PER slightly differently: multiple-choice single response (MCSR), MCMR, short answer, ranking tasks, Likert scale, and free response. Table I summarizes the connections between types of questions common to PER and the types of quantitative data they provide that we argue for below.

\section{Multiple choice}

Probably the easiest kind of question to administer and with which to collect data is the multiple-choice question [19]. Especially when using a bubble-sheet scanner system or computer-based administration, the recording and entry of the data can easily be automated, and even when it is not, it is still easy to record the data (though perhaps more time consuming). Indeed, one of the most commonly used written surveys in PER, the FCI, makes exclusive use of multiple-choice questions. However, not all multiplechoice questions are created equal, and thus one must distinguish between different varieties of multiple-choice questions.

Single response.-A MCSR question asks students to select one response out of a given set. Each response is mutually exclusive of the others, and their order of presentation is mostly independent of each other. For these kind of questions, each question requires a separate coding dimension into which the student's answer is placed. Each of these coding categories can be considered a single data point, but in order to determine the appropriate data type for these points one must know whether the students are given an opt-out response to the question, i.e., none of the above or a similar choice. If an opt-out is provided, then the answers are coded as asymmetric nominal data. The optout response does not provide any definitive information about the answer the students actually want to give; it just indicates that they did not like any of the other responses. For example, multiple-choice questions on the FMCE have the option "J. None is correct" and so should be encoded as asymmetric nominal data [20]. On the other hand, answers to questions without an opt-out response, such as most of those on the FCI, can be coded as symmetric nominal data, since each of the responses does contain an affirmative statement. Of course, students may elect to opt out of a 
question by simply not answering it, but that does not necessarily lead to encoding the answers as asymmetric nominal data: missing data are not the same as an opt-out response. For a more detailed discussion of missing data, refer back to Sec. II C.

Figure 2 shows an example of a MCSR question. There are five possible responses, each of which expresses a different idea about how the drop times between the two balls are related. As such, a symmetric nominal scheme with five states would be the most appropriate for encoding the students' answers to this question. Indeed, representing each answer by its respective letter choice is the obvious encoding scheme, though the use of other arbitrary symbols is equally valid. Using numbers is possible, but carries with it a special consideration. Numbers have well-defined addition and multiplication properties (they are a form of ratio data), e.g., the distance from 1 to 3 is the same as the distance from 3 to 5 (i.e., two units), while the distance between nominal data states cannot be defined (only the fact that they are different is available). Once disconnected from the original instrument, a table of numbers suggests that differences between students can be measured with subtraction based tools or that an "average response" can be calculated. Neither of these is appropriate, a fact that is easy to see when the states are represented by letters: $(\mathrm{D}-\mathrm{A})$ and $(\mathrm{A}+\mathrm{D}+\mathrm{C}+\mathrm{D}) / 4$ are both meaningless. As a result, when using numbers, the researcher must be careful to keep the original context in mind and not conflate the properties of numbers per se with the properties of the data the numbers are being used to represent.

Multiple response.-A MCMR question provides the students with a set of responses to a question and asks them to pick out the ones that are correct. Any one or even more than one of the responses may be correct and students may choose as many as they want. Because it is not clear whether a student did not choose a response because it was wrong, because they were not sure about it, or because they felt their other responses constituted a sufficient answer, each response has an implicit opt-out option built into it. As a result, asymmetric nominal encoding is appropriate in this case. However, the number of data points and the number of states for each point depends on some additional decisions. At one end of the spectrum, each answer option should be coded separately, giving $N$ codes with two states each: chosen and not chosen $(N$ being the number of answer options). Since each option is coded separately, it is possible for students to have partial matches to each other when they choose some of the same responses, but not an identical set of responses (e.g., one student might pick $\mathrm{A}, \mathrm{B}$, and $\mathrm{D}$ while the other picks $\mathrm{B}, \mathrm{D}$, and $\mathrm{E})$. This emphasizes the similarities between the them. At the other end of the spectrum, answer options are grouped together (generally by question) to create one code with $2^{N}$ states. In this case, each set of responses is its own answer state and partial matches are not allowed (this might be useful if a partial match is considered to be a false positive). Given these two options, choice of representation of the codes is important because some choices will allow you to go back and forth between the options (see our example below) while others will not.

Figure 3 shows an example of a set of MCMR questions. In this case, each question has a set of 7 points from which the students must pick as few as 0 or as many as 7 as correctly answering the question. Thus each question has $2^{7}$ possible responses. While there are many possibilities for representing these answers, our suggestion would be to represent each answer possibility as a sequence of 7 binary numbers. In this case 0000000 would correspond to an answer of "none" by the student, "0100101" would correspond to selection of choices $\mathrm{B}, \mathrm{E}$, and $\mathrm{G}$, and an

1. Two metal balls are the same size, but one weighs twice as much as the other. The balls are dropped from the top of a two story building at the same instant of time. The time it takes the balls to reach the ground below will be:

(a) about half as long for the heavier ball.

(b) about half as long for the lighter ball.

(c) about the same time for both balls.

(d) considerably less for the heavier ball, but not necessarily half as long.

(e) considerably less for the lighter ball, but not necessarily half as long.

FIG. 2. An example MCSR question from Hestenes et al. [21]. Data are symmetric nominal. 
ET3-LMCT6: POTENTIAL vS POSITION GRAPH II-FORCE

Shown is a graph of electric potential versus position for a region of space. The labeled points indicate seven locations within this region.

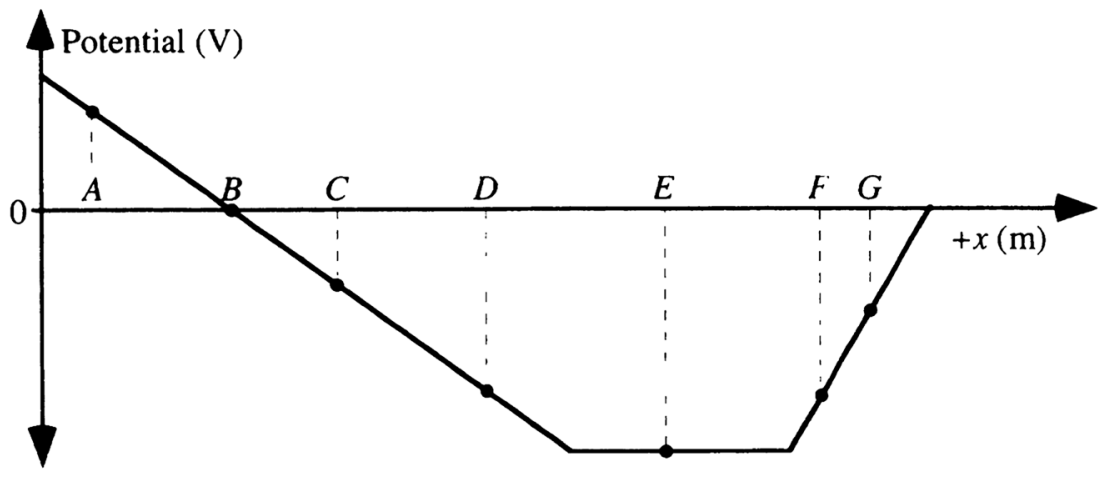

Use one or more of the letters for the labeled locations in answering the questions, or write "none."

1) At which location(s) would a positive charge have the largest force on it?

2) At which location(s) would a negative charge have no force on it?

3) At which, if any, of the location(s) is the electric field zero?

4) At which location(s), if any, is the electric field in the same direction as at $A$ ?

5) At which location(s), if any, is the electric field in the same direction as at $B$ ?

6) At which location(s), if any, does the electric field have the same magnitude field as it does at $C$ ?

FIG. 3. An example MCMR question from Hieggelke et al. [22], for which response data are asymmetric nominal.

answer of "1111111" would correspond to a selection of all possible responses, i.e., "A, B, C, D, E, F, G," by the student. This scheme has the advantage of making the selection of whether to emphasize similarities or differences one that is easily changed. To emphasize similarities, one can treat each bit in the sequence as a separate dimension; to emphasize differences, one can treat the entire sequence as a single dimension.

The real difficulty in dealing with multiple-choice multiple-response (MCMR) questions comes in dealing with the all-null state. If the question has an explicit optout response (which should not be coded as a separate dimension, see Ref. [23] for an example), or some other mechanism for ensuring that the student considered the question and did not pick any of the responses as right (such as the instruction to write none in the example cited above), then the all-null state is straightforward to encode (in our binary sequence example, 0000000 is the all-null response). However, if the question lacks these mechanisms then it is impossible to tell the difference between the allnull state and missing data (i.e., the question was skipped and our data table should have a blank in it, not a 0000000). This forces the researcher to make some assumptions about the students and decide whether skipping or a true all-null response is more likely.
As with multiple-choice single-response (MCSR), the researcher has to be careful when representing MCMR questions with numbers not to conflate the properties of numbers per se with those of the data they are being used to represent. Our examples above could just as reasonably have used "o" and "•" instead of " 0 " and " 1 " to represent the student responses.

\section{Short answer}

Like multiple-choice questions, short-answer questions tend to be easy to administer on a large scale [19]. While they might appear to be free-response questions at first glance, short answer questions are generally phrased in such way that creates the expectation of a quick answer. As a result, students' answers are generally no longer than a single phrase and thus relatively unambiguous. Since student responses are not constrained to a given set of responses, however, the number of possible responses that a student could give is effectively infinite (though in practice there is probably a much smaller set of responses that are actually given). As a result, the coding choice is not always obvious.

If the answer to a short-answer question takes the form of a number, then the responses can be encoded as interval or 
ratio data very easily. Doing so allows for rounding errors in student math (as they generally result in very small differences) but does not really look at the underlying mathematical processes that a student is using. If one student uses $2 \mathrm{~m} / \mathrm{s}^{2} \times 2 \mathrm{~kg}=4 N$ and another uses $2 \mathrm{~m} / \mathrm{s}^{2}+2 \mathrm{~kg}=4 N$ both students' answers $(4 N)$ would be considered identical as interval or ratio data. Physically, however, the first response may be appropriate, while the second is absurd. In this case, it may be appropriate to categorize the responses into groups based on the mathematics used to arrive at the answer. In this case, the encoding would likely be as asymmetric nominal data, as it is likely that this encoding will require an "other" category.

For short-answer questions that are answered with a word or phrase, it is generally necessary to categorize the responses somehow, thus leading to encoding as asymmetric nominal data (asymmetric because of the ubiquitous other category). Care, of course, should be exercised when doing so to introduce as little interpretation of the student response as possible, as any interpretation in the encoding process will be reinforced in the analysis and thus appear more significant than it actually is.

Figure 4 gives two examples of short-answer questions. In this case different strategies might be used for encoding each question. For the first question it would make sense to create a list of the forces that students list and then encode for each student which of those forces they gave. In this case we are essentially treating the question as a MCMR question for which the possible responses were not given ahead of time. The second question, on the other hand, might be encoded numerically as ratio data, but given that magnitudes for the spring constant and the initial velocities are not given and that only the relative magnitude of the masses is given, there is a fairly good possibility that some students will be unable to arrive at a numerical answer (despite the fact that the correct answer is 0). As such, it may make more sense to categorize the responses as "zero," "containing internal forces," or other (i.e., as a single asymmetric nominal source).

\section{Ranking tasks}

Popularized by Maloney [25], ranking tasks provide students with a single physical scenario and a set of variations on one or two of the physical parameters. Students are then asked to rank the variations based on another physical parameter that is not given, but that can be determined based on the given information. Since students are being asked to rank the situations, we are dealing with ordinal data: specifically a within-students ranking. Encoding the response involves recording the rank for each item and thus requires as many codes as there are items being ranked. Also, since students are likely unaware of the considerations involved in dealing with ties in ordinal data, the encoding of ranking task data will require enforcing the mid-rank method on a student's ordering.

Figure 5 shows an example of a ranking task. Others can be found in Maloney [25], Friedel [26], and O'Kuma et al. [27]. In answering these questions, the student holds the rank order constant and "moves" the letters around to achieve their answer. However, to encode their response, it is easer to do the opposite: hold the item order constant and "move" the rank values around to represent the student's response (while also correcting for the handling of ties). As a result, the correct answer, 1: $\mathrm{A}$ and $\mathrm{D}$ and $\mathrm{F} 4$ : $\mathrm{B}$ and E 6: C might be coded as A: 2, B: 4.5, C: 6, D: 2, E: 4.5, F: 2. (Note the application of the mid-rank method to deal with the three-way tie for the first place and the two-way tie for the fourth.) In a tabular representation of multiple student responses, we would probably also drop the letters from the coding and instead use them as column headers with each column representing a different item.

One thing to bear in mind when coding ranking tasks, however, is that each ranking task involves a separate ranking. Many analysis methods for dealing with ordinal

III. Application of momentum conservation to two-dimensional collisions

The spring formerly connecting blocks A and B is disconnected from block $B$. The blocks are given initial velocities in the directions shown so that they will collide with the spring between them. As in section II, system C refers to the combination of both blocks and spring.
A. What are the external forces exerted on system $\mathrm{C}$ during the collision?

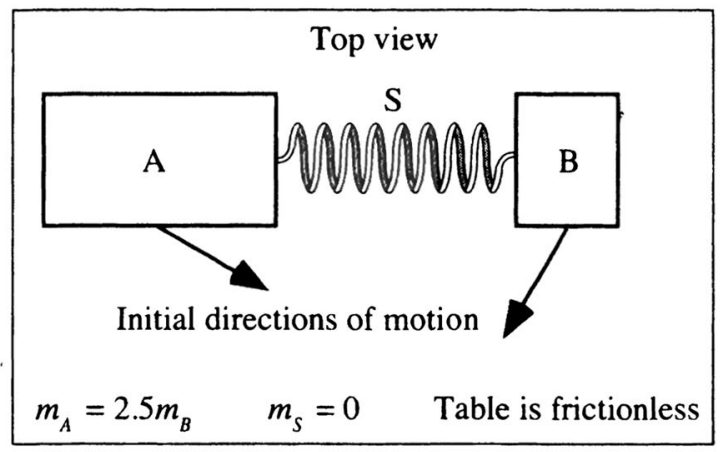

What is the net force on system C?

FIG. 4. An example of a short-answer question from McDermott et al. [24]. Data can be coded in various forms, though asymmetric nominal is the most common. 


\section{Ball Motion Diagrams-Velocity I ${ }^{1}$}

The following drawings indicate the motion of a ball subject to one or more forces on various surfaces from left to right. Each circle represents the position of the ball at succeeding instants of time. Each timeinterval between successive positions is equal.

Rank each case from the highest to the lowest velocity based on the ball's last velocity using the coordinate system specified by the dashed arrows in the figures. Note: Zero is greater than negative, and ties are possible.

A

B

C
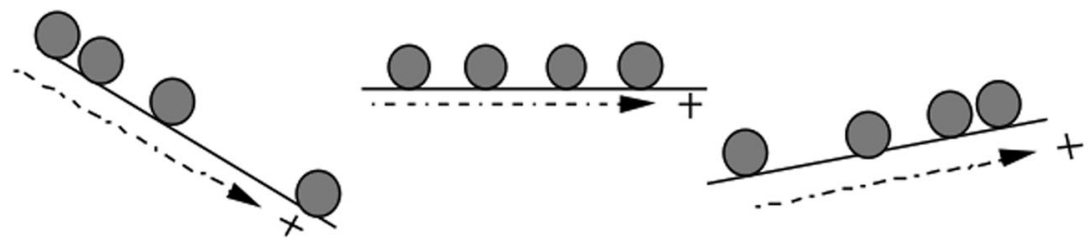

D

E

$\mathbf{F}$
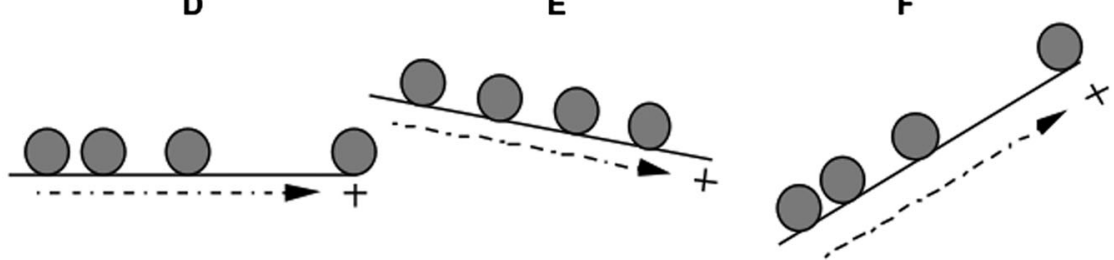

Highest 1 2 3 4 5 6 Lowest

Or, all have the same velocity.

Please carefully explain your reasoning.

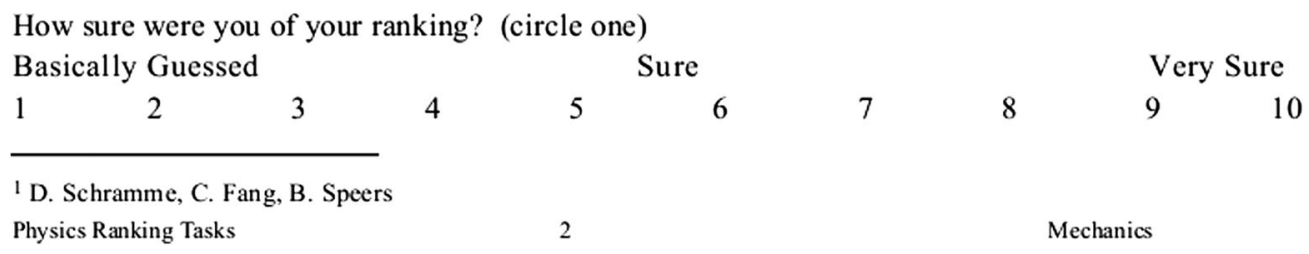

FIG. 5. An example of a ranking task from O'Kuma et al. [27]. Data are coded as ordinal.

data assume a single ranking. As a result, the researcher must exercise considerable care in situations where multiple ranking tasks have been assigned to a student. In these instances, it will often be necessary to repeat some part of the analysis for each ranking task assigned and then devise a method of combining those intermediate results in order to continue the analysis.

\section{Likert scale}

On a Likert-scale question, students are asked to rank themselves for each prompt on a set point scale (usually 5 or 7) relative to agreement, confidence, or some other criteria. One example can be see at the bottom of Fig. 5 where students are asked to assess their confidence level in their answer on a 10-point scale. For an example that makes 
Here are 34 statements which may or may not describe your beliefs about this course. You are asked to rate each statement by circling a number between 1 and 5 where the numbers mean the following:

\begin{tabular}{|l|l|l|l|l|l|}
\hline 1: Strongly Disagree & 2: Disagree & 3: Neutral & 4: Agree & 5: Strongly Agree \\
\hline
\end{tabular}

Answer the questions by circling the number that best expresses your feeling. Work quickly.

Don't over-elaborate the meaning of each statement. They are meant to be taken as

straightforward and simple. If you don't understand a statement, leave it blank. If you

understand, but have no strong opinion, circle 3. If an item combines two statements and you

disagree with either one, choose 1 or 2.

1 All I need to do to understand most of the basic ideas in this course is just read 122345 the text, work most of the problems, and/or pay close attention in class.

FIG. 6. An example of a Likert-scale item from Redish et al. [28]. Data are coded as ordinal.

exclusive use of Likert-scale items, the Maryland Physics Expectations survey (MPEX) ask students to rank themselves on a 5-point scale of agreement with each prompt [28] (see Fig. 6). Since ranking is involved, responses should be encoded as ordinal data, but whether it is withinor between-students ordinal data depends on the point of view.

The point of view that leads to within-student ordinal data assumes that each student considers all 34 statements as a single unit (though not necessarily simultaneously). This means that if a student answers 5 (Strongly Agree) to one statement and 4 (Agree) to another, then we can conclude that particular student agrees with the first statement more than the second. This allows us to determine the student's effective ranking of the statements based on their agreement level (albeit, with many ties since there are just 5 ranks and 34 statements).

The point of view leading to between-student ordinal data assumes that each item considers all the students as a single unit (clearly not simultaneously). This means that if one student chooses 1 (Strongly Disagree) for a statement and another chooses 2 (Disagree) for that same statement that the first student more strongly disagrees with that statement than the second.

Both of these points of view have their problems. In the case of the former, not all students use all the available ranks. This implies extra "space" between their answers that a simple assumption of ordinal ranking does not capture. In the case of the latter, there is no guarantee that the students are treating the scale in the same way. The difference between Strongly Agree and Agree for one student may be different than for another. To put it in more scientific terms, the students' scales are not calibrated against each other.

In either case, there will likely be many tied ranks. As a result, it will probably be necessary to re-rank the answers in preparation for analysis if the number of ranks used or the "average rank" (i.e., the rank all items would get if there was no way to tell the difference between them) makes a difference to the measure being used. However, the ease with which new data are added to the data set after such a reranking has been done is dependent on the choice between within- or between-students ranking and the kind of data being added: within-students ordinal data make the addition of more students easier than the addition of new questions, while between-students ordinal data makes the reverse true. Done this way, new data can be simply inserted into the existing ranking scheme. When done the other way around, new data require redoing the whole re-ranking process. As a result, researchers need to weigh the benefit of ease with the problems indicated above.

In the example prompt in Fig. 6, a first-pass encoding of the question would simply be the number that the student circled. Once that has been done for all students for all prompts it then becomes possible to re-rank the answers by prompt or student so that the ranks follow the tying conventions discussed earlier (see Sec. II B). Given that the MPEX has a fixed form (i.e., we do not expect the addition of new prompts to the survey), but we might administer the survey to additional classes in the future and want to compare their results to the current data set, it would make adding that potential data in the future easier if we re-rank by student to create a within-students ordinal ranking. Additionally, since it is considered "favorable" to agree 
with some items on the MPEX and "unfavorable" to agree with others, we may want to rank according to the favorable response on each item, rather than agreement, though this is not necessary to compare students to each other.

Even if the researcher decides to use some other encoding scheme, there are some cautions (suggested by the problems noted above) that should still be borne in mind. A major caution is the tendency to want to look at a "class average" for a particular prompt as a way of characterizing the whole group. The default average function for most physicists is the arithmetic mean, which is dependent on being able to add data points together. Ordinal data, however, does not have a well-defined addition function; only distinctness and order are well defined. In such a situation, either the mode (whose calculation depends on distinctness) or the median (whose calculation depends on order) would be much more appropriate for computing the average rank (and in which case the terms "mode" or "median" should probably be favored over "average" in order to make clear that the mean is not being used).

\section{Free response}

The most difficult type of question to deal with is the free-response question. At the same time, however, it is a fairly common type of question for PER written surveys to use, particularly in the form of a request to "Explain your reasoning." Free-response questions place almost no restraints on student answers other than that they answer the question, though often it is unclear whether the answer completely maps the space of student thinking [23], since student beliefs as to what constitutes a sufficient answer may differ from what the instrument designer intended. As a result of this lack of constraints, the data type of a typical free-response question code is not determined by the question itself, but by how the answers are graded or coded. Point grades, for instance, would constitute encoding as interval or ratio data, while a rubric grade is encoded as between-students ordinal data. Answers could also be grouped into categories, thus encoding as nominal data (symmetric if you create meaningful categories to cover all answers, asymmetric if not). One could also code for the presence or absence of certain features such as picture components or certain key words (and their synonyms), creating another possible way to encode as asymmetric nominal data. If answers are largely or exclusively text, then the techniques of latent semantic analysis can generate codes which are ratio [29]. The choice of coding scheme also influences the number of dimensions that will come from a single question (and whether these dimensions are independent or not). Point or rubric grades generally result in only one dimension, possibly even less than one (when multiple questions are combined to create a single score), while categorical or feature-based coding will result in many.
Because of the immense variety of how a set of freeresponse questions can be treated, it is impossible to give any specific guidelines. The only thing that we can say is that the researcher should carefully consider and justify each step of their encoding process so that other researchers reviewing their work can assess for themselves how accurately the encoding represents the data and not the original researcher's biases.

\section{B. Matching to analysis methods}

As we mentioned above, several methods of analysis start by computing the difference (or similarity) between each data point. As a result, they are amenable to use on all sorts of data, provided the difference (or similarity) is calculated in a manner which is appropriate to the type of data under consideration. Mathematically, this calculation is isomorphic to determining the distance (or correlation) between points in the arbitrary space defined by the test used to elicit those responses. Each point in the space represents one possible set of responses to that test. The more similar these responses are, the closer these points should be; the more different, the further apart. In order to ensure this, one must take into account the properties of the data taken when defining the space, i.e., the axes and the values available on those axes. Otherwise, any mathematically based analysis will lack a firm foundation. While calculating a distance or a correlation may seem like two different tasks, in practice they are simply approaching the same problem from different starting points. Given a distance between two points, it is possible to define a corresponding correlation, and given their correlation it is possible to define a corresponding distance between them. Our discussion below of some of the possibilities will thus switch back and forth between distances and correlations according to the original use of the measure. For a detailed discussion of how to calculate a particular measure, including how to go from a correlation to a distance measure or vice versa, see Springuel [30].

\section{Minkowski distances}

Minkowski distances are a class of distances which includes the Euclidian distance with which most physicists are familiar [2-5]. In calculating a Minkowski distance, it is necessary to find the difference (using the mathematical definition) between two states. As a result, addition must be a well-defined process for the data type and so Minkowski distances should only be used on interval or ratio data.

\section{Simple matching coefficient}

The simple matching coefficient (and its variants) is a correlation based on a count of the number of dimensions on which two data points are in the same state $[2-5,31,32]$. It assumes that all states are equally informative, i.e., all states are equal to themselves and not equal to all others. 
This requires that the data have the property of distinctness well defined for all states, suggesting that it is most appropriate for symmetric nominal data, though in some circumstances it might also be used with interval or ratio data (where distinctness is also well defined). We would not recommend this, however, since the number of states for such data are theoretically infinite, which means that the simple matching coefficient will tend towards 0 as the instrument used to collect the data gets more precise.

\section{Jaccard coefficient}

Like the simple matching coefficient, the Jaccard coefficent (and its variants) is a correlation based on a count of the number of dimensions on which two data points are in the same state $[31,33,34]$. However, it also assumes the existence of a null state (i.e., a state which is uneventful) and does not count matches of this state as either equal or not equal. This makes it appropriate for asymmetric nominal data, where such a null state exists. It could also be used for symmetric nominal data which has holes in the data. In this case the "missing" data would be the null state. Usage with interval or ratio data suffers from the same problem as indicated for the simple matching coefficient.

\section{Pearson correlation coefficient}

This measure (and variants based on it) treat the two data points as the two halves of a set of paired points [35]. It then calculates the strength of the linear relationship between those two lists. This requires that each dimension of each data point be on a similar measurement scale and that it be possible to calculate averages and standard deviations. As a result, it is most appropriate for interval or ratio data, especially when such data has been normalized, or otherwise scaled to a consistent standard.

\section{Spearman rank correlation}

Based on the same mathematical tools as the Pearson correlation coefficient, the Spearman rank correlation is less sensitive to outliers and more sensitive to monotonic but nonlinear relations than the Pearson correlation coefficient $[35,36]$. It achieves this by first ranking the data within each list before calculating the correlation coefficient. It would thus seem most appropriate for ordinal data, which is naturally ranked this way, or for interval or ratio data, which can have such a ranking derived from it. However, because it uses the mathematical tools of the Pearson correlation coefficient, in particular the arithmetic mean and standard deviation, it does not really treat the ranking (whether natural or derived) as true ordinal data. Rather it treats it as if it had interval properties. As a result, it would be best to use the Spearman rank correlation only when its decreased sensitivity to outliers and increased sensitivity to monotonic, nonlinear relations is considered to be of sufficient benefit to overcome the inherent defect of not respecting the properties of the data.

\section{Kendall's $\tau$}

Like the Spearman rank distance, the Kendall's $\tau$ distance requires that the data be ranked as the first step in the analysis (and thus is appropriate for data in which order is well defined: namely, ordinal, interval, and ratio) [16]. It then, however, compares relative order between items in two different rankings, looking for either a concordance or discordance of rank order (though not necessarily ranks). This frees Kendall's $\tau$ from the dependence on the concepts of mean rank and standard deviation of ranks, which the Spearman rank correlation has (because of its use of the mathematical tools of the Pearson correlation coefficient). This makes Kendall's $\tau$ far less problematic to use with ordinal data. It is also slightly less problematic to use it for interval or ratio data, but the process of ranking that data is a loss of information and so should be justified by some expected benefit.

\section{A REVIEW OF PRIOR RESEARCH}

Given the necessarily abstract nature of the above theoretical discussion, we now turn our attention to concrete cases of research in order to highlight how data encoding has been treated thus far in PER. In many cases, including all of the ones we discuss below, the encoding process has not featured prominently in the papers using a particular research method. As a result, we reconstruct the encoding process involved as best we can. We point out those places where we think that the encoding process could be improved as well as those places where we think that it has been done well. Since we cannot hope to be exhaustive in this discussion, we focus on the most prominent quantitative research methods in PER: normalized gain, factor analysis, model analysis, cluster analysis, and epistemological agreement.

\section{A. Normalized gain}

While not original to PER $[37,38]$, the normalized gain has become a fairly standard metric for use with multiple choice tests, particularly the FMCE and FCI since its introduction to the field [39]. Despite the variety of situations in which it is used, the encoding procedure used with normalized gain is largely identical and fairly straightforward, and therefore rarely mentioned in the literature.

The calculation of the normalized gain is based on the comparison of two scores on the same instrument taken at different times, usually before and after instruction. With this in mind, the encoding process used when the normalized gain is to be calculated is geared towards the generation of scores. Since in most cases the score is simply the percentage of questions answered correctly, a simple binary encoding is used: for each question a student's answer is 
marked "right" or "wrong" (which might be represented numerically as " 1 " and " 0 ").

The most significant deviation from this simple encoding practice is to weight and/or combine questions. We can see both of these deviations in use on the FMCE. On that test, several questions (e.g., question 15) are omitted from the calculation of the score (given a weight of 0 ) because they are considered to test something other than the physics thinking of the students [20]. Other questions (e.g., 8, 9, and 10) are combined so that a student must answer all of them correctly in order to get credit for a right answer. This serves to insulate the score from false positives that might arise on certain questions in these groups [20,40]. Additionally, these deviations may be combined, as in a commonly used analysis [40], where several sets of three questions $(8,9$, and 10;11, 12, and 13; and 27, 28, and 29) are scored as a unit, which is given a weight of 2 .

While well matched to the analysis method, the encoding scheme described above is not very well matched to the questions that the students were asked and therefore to the responses they gave. Neither the FMCE nor the FCI (nor any other multiple-choice instrument in use in PER that the current authors are aware of) presents the students with just two options: a right answer and a wrong one. In general, there are several wrong answers for each question (as many as 8 on FMCE questions 14-21). Mapping all of these answers into a single wrong encoding represents a significant loss of information. This is especially true when these wrong answers include well-designed distractors and are not chosen at random. Ideally, in order to match the encoding scheme to the questions, information about which answer a student choses, whether it be right or wrong, should be retained. Beyond this basic indication, we really need to look at the test itself to make more specific recommendations. We do this below for the FCI and FMCE as examples.

To start with, we consider the FCI. It consists of 29 multiple-choice questions and student answers to each question fall into one of 5 categories (4 for question 16). Based purely on the answers, then, it is possible to tell whether two students have the same or different answers for a particular question, that is, the answers to each question are generally distinct. Furthermore, there is nothing inherent to the test itself that indicates a preferential order between the options. One could be introduced from an outside source (such as an evaluation of how right or sophisticated an answer might be) but this is not present within the answers themselves. This indicates that the data from each question should be considered either asymmetric or symmetric nominal, depending on whether or not a none of the above option is available for that question. Of the 29 questions, four (numbers 5, 12, 15, and 21) have a variant of none of the above for the last option. However, in three cases $(5,12$, and 21), these variants include additional information which specifies them enough that students who chose these options have actually affirmed a response despite the none of the above phrasing which starts the answer. Only in one case (question 15) is the option simply none of the above. In this case the student has only negated the other responses; they have not affirmed any response. As a result, the answers to this question should be classified as asymmetric nominal, while the answers to all the rest can be classified as symmetric nominal data.

Looking at the FMCE, it consists of 43 multiple-choice single-response questions (47 if the energy cluster questions are included) [20,41]. By and large these questions have six or more responses, with sets of responses being shared by at least three questions (question 39 and the energy cluster questions being the notable exceptions). One thing that all the questions share, however, is that they all include an option "J. None of the above" (with some variation in the actual wording). This means that unlike the FCI, where most of the questions provided symmetric nominal data, the questions on the FMCE all provide asymmetric nominal data.

In the current authors' experience, there is one circumstance in which the encoding scheme is better matched to the questions. When the multiple-choice test is given with a bubble response sheet and the computer scanning these sheets is not given a key to indicate which answers are right and which are wrong, then it defaults to simply reporting the letter of the answer the student gave to each question. Indeed, the spreadsheet analysis tool developed by one of the current authors (MCW) for analyzing the FMCE relies on this sort of encoding [42]. This better match of the encoding to the question, however, also leads to a worse match between the encoding and the analysis method. Even if we deliberately encode all the answers by letter, the first step of an analysis using a normalized gain will still require the calculation of a score and thus the loss of information about the nature of the wrong answers given. There is no perfect match between question, encoding scheme, and data analysis in this instance. This does not mean that there is anything wrong with using the normalized gain, just that there is a limitation that ought to be acknowledged. Considering how the encoding scheme matches to the questions and the data analysis helps to highlight this fact. We note that there have been some efforts to reduce this mismatch by computing additional scores for alternative student models and then calculating the normalized loss associated with those alternative models $[43,44]$.

Another issue that can come up with the normalized gain is the question of when (during the analysis) to take the average. Bao [45] has shown that the choice of timing can have significant effects on the normalized gain in certain circumstances and that the size and direction of these effects might actually say something about the way the class has responded to instruction. While this issue does not appear immediately pertinent to the encoding, the choice of encoding changes how easy or hard it is to generate scores 
and thus take their average at various points in the analysis process. For instance, the " $0-1$ " encoding which is typically used makes the generation of scores very early in the analysis very easy. Indeed, it is possible to generate those scores in wide variety of ways, including some that Bao [45] did not consider (such as by item). On the other hand, when the answers are encoded as letters so that one can retain as much information as long as possible, generating those early scores is harder [46], and therefore less likely to occur. As a result, it would be useful to consider not just the kind of analysis to be performed, but the intended order of the steps when devising an encoding scheme.

\section{B. Factor analysis}

While the researchers designing a test often have particular relations between the questions in mind, there is often little evidence that the students perceive the same relations. Factor analysis attempts to address this by looking at the correlations in the way that students answer questions. There have been several instances of applying factor analysis to multiple-choice surveys in PER (e.g., Refs. [47-49]). These instances have applied factor analysis to a variety of different tests, but all have used the same encoding strategy: each answer is encoded as either right, in which case it is given a value of 1 , or wrong, in which case it is given a value of 0 . Furthermore, each question had just one right answer and the rest were considered wrong. Specifically in the context of the FCI, Hestenes and Halloun have criticized this basis for factor analysis because it fails to account for the student concepts of force [50]. Only the appropriate Newtonian concept gives a clear signal in this coding scheme. All other student concepts of force are lumped together as merely wrong. More generally, we also note that this is the same encoding scheme as we described above for the normalized gain analysis, where the same concerns (and suggestions) about how well the encoding scheme matches to questions of the FCI and FMCE apply.

In a separate example, Ding and Beichner used the Matter \& Interactions Energy Test (MIET) with this encoding scheme [49]. While we can quickly say that we have the same general concerns that apply to encoding multiple choice questions into a right or wrong scheme, we need to look specifically at the test itself to come up with more specific recommendations. The MIET consists of 33 multiple-choice single-response questions (originally numbered 1-28, with numbers 3,6 , and 16 having two parts each and number 10 having three parts) [51]. Each question has between four and seven possible responses for the students to choose from. Of these questions, eight have a none of the above option (questions 2, 4, 10a, 10b, 10c, 14, 18 , and 21) and nine have a "not enough information" option (questions 6a, 8, 9, 11, 16a, 16b, 23, 26, and 28). Not enough information and none of the above are distinct enough ideas that both responses could appear in the same question: none of the above implies that the correct answer is not present in the other available answers, while not enough information implies that the question cannot distinguish between some or all of the other available answers. However, on the MIET they do not co-occur and thus both can be treated as a negation of the other available responses by a student, rather than an affirmation of any one idea. This implies that the answers to these questions should be coded as asymmetric nominal data. For the remainder of the questions (questions 1, 3, 5, 6b, 7, 12, 13, 15, 17, 19, 20, $22,24,25$, and 27), there is no explicit opt-out response provided for the students and so these responses should be coded as symmetric nominal data [52].

A potentially larger issue, however, is how this encoding scheme matches to factor analysis. Factor analysis analyzes the correlations between the encoded data on various questions. As such, computing the correlation between the student-provided answers on each question in a pairwise fashion is the first step. Huffman and Heller [48], Ramlo [47], and Ref. [49] then make use of the Pearson correlation coefficient (also called a phi coefficient in this case since the data are binary) for each pair of questions as a measure of their relatedness. However, the authors do not address the fact that for binary data, the Pearson correlation coefficient cannot reach its most extreme values $(-1$ and 1$)$ unless the row totals are equal to each other and the column totals are equal to each other [53]. This means that each question must have the same number of students getting it correct in order for the Pearson correlation coefficient to be a valid choice under the right or wrong dichotomization of the data being used [54]. However, Hestenes et al. [21] showed that the success rate on different questions was different in their validation of the FCI, Thornton and Sokoloff [20] showed this for the FMCE, and Ding [51] did the same for the MIET. Since Ramlo [47], Huffman and Heller [48], and Ding and Beichner [49] did not show that the data they subjected to factor analysis differed from the original data set in this respect, it is probable that they all would run into this problem. The Pearson correlation coefficient, therefore, is a questionable choice for determining the correlation between two questions on these tests.

A better choice, if the dichotomous data are to be used, would be the tetrachoric correlation [53]. Alternatively, rather than encoding the data into dichotomous information, one could also use the original number of options for each item. In this case, either the simple matching coefficient or the Jaccard coefficient would be more appropriate. The choice could be determined by whether or not the items being correlated had a none of the above option. Indeed, on the FCI and MIET, which have questions both with and without the none of the above option, it would probably be necessary to use both types, which introduces its own set of complications. Additionally, care would be needed in identifying wrong answers that are consistent (and thus should be considered a match) and those that are 
not consistent in order to properly address the concerns raised by Hestenes and Halloun.

\section{Model analysis}

That students can have multiple ways of interpreting physical phenomena and may use different methods of interpretation in different situations has been commonly acknowledged by those designing tests like the FCI and FMCE $[20,21]$. Model analysis attempts to capture this phenomena by comparing student responses to a welldesigned multiple-choice instrument (like the FCI and FMCE) to not only the correct model, but also "a small number of strong naive conceptions." [56]. With these multiple comparisons, one can estimate the probability that a student will use each of these models and then combine these probability vectors to create a density matrix which represents not just how many students followed each model, but also how confused the students were about the models (defined, by Bao and Redish, as choosing answers consistent with multiple models over the course of the instrument).

Since model analysis compares each individual student to multiple models and retains information about each of those models throughout the analysis, its encoding scheme must reflect those multiple models. In model analysis, the right or wrong dichotomy used as the basic encoding for normalized gain and factor analysis is generally insufficient. A more complicated mapping is needed in which each answer is assigned to one (or more) of the known models being examined or to an other category. This mapping can get fairly complicated as multiple answers may be assigned to the same model (e.g., in Bao and Redish, question 5 has three answers assigned to model 2), multiple models may be assigned to the same answer (e.g., in Smith and Wittmann [40], answer $b$ is assigned to both the mass dependence and action dependence for question 38), and the model may only be determinable by combining the answers from several questions (e.g., in Smith and Wittmann [40], the reversing direction cluster). It could also be quite simple, with the right or wrong dichotomy being the limiting case (just 1 model to which just 1 answer corresponds, with everything else as other). However, in either case it is constrained by the number of models which the researcher knows about and is prepared to encode for in advance. In published work using model analysis, this seems to be limited to 3 models (the NewtonIII cluster in Smith and Wittmann [40]), but mostly involves 2 (not counting other). This mapping is then represented by a vector with a number of dimensions equal to the number of models (including other) and a value of 0 or 1 for each dimension to indicate whether the student's answer agreed with that model or not on that question.

The encoding scheme just described is very well matched to the methods of model analysis. The first step is a fairly straightforward average of the vectors for each student, yielding a vector that represents the probability that the student uses a given model. This match should not be surprising, as the encoding scheme was almost certainly designed with model analysis in mind. Now, while one could argue that averaging, a process dependent on the addition of data states, is not well matched to the asymmetric nominal coding (essentially "yes" and "no" for whether a particular answer matches with a particular model), the fact that the average is understood (and treated) as a probability renders this criticism far less potent. This might be easier to see if one thinks about it in terms of coin flips. While it does not make sense to say the average of some number of coin flips is some value between heads and tails, it does make sense to say that based on those coin flips, the probability of getting heads when flipping that coin is some value between 0 and 1 ( 0.5 if the coin is fair).

As for the matching of the encoding scheme to the data, the inclusion of multiple models means that this match is better than the right or wrong dichotomy that both normalized gain and factor analysis used because more of the diversity of the student responses is being maintained. Furthermore, insomuch as tests like the FCI [56], the FMCE [57], and the MIET [49] are designed to have meaningful distractors, i.e., some wrong answers are meant to represent and trigger certain models of student thinking, the matching between an answer and a model is very much in line with what the tests are supposed to be doing. It is, of course, not perfect. Students may pick an answer for some other reason than that they were using the model it is supposed to represent (this is probably most obvious when an answer is supported by multiple models), but this sort of confounding factor is why error bars are necessary [57]. From an encoding standpoint, the more interesting difficulty comes from the other category. Since model analysis is looking exclusively for agreement with known models, a set which is usually very small in number, each question usually has multiple answers that are classified as other. While this is probably acceptable for answers that were added to the test in order to round out the answer set, for answers that are meant to represent a particular student model observed during the test design, or that have come to be associated with such a model in a post hoc fashion, classifying them as other would be inappropriate. It should be noted that we know of no example where this overuse of other has happened, so we include this warning purely as a cautionary statement.

\section{Cluster analysis}

While the above discussed methods of analysis are aimed at saying something about a predefined group of students (the whole data set, a particular class, classes taught using particular methods, a demographic group such as female students, etc.), cluster analysis aims to break the data set up into groups of students who are "similar" to others within the same group and "different" from those in other groups. 
These groups can then be used in a variety of ways ranging from simply characterizing the behavior of the students in the data set to laying the ground work for future research using one of the above discussed methods. Regardless of what is done with the results, however, the way that the data are encoded makes a very big difference as to which students are considered similar to each other and therefore are in the same group. To demonstrate this, we will look at two papers that make use of cluster analysis in conjunction with a multiple-choice instrument, but with vastly different encoding methods.

In the first paper, Ding and Beichner [49] looked at grouping students based on their performance on the MIET. To do this, Ding and Beichner split the MIET up into five topic areas representing between 2 and 15 questions [51]. In each of these areas, students are given a score representing the percentage of the questions on that topic that they answered correctly. As discussed above, scoring the questions leads to a mismatch between the sorts of responses given to the questions (multiple-choice questions are more appropriately encoding using a nominal scheme). On the other hand, the generated scores (which have interval properties) are then well matched to the Euclidean distance, which Ding and Beichner used to measure the difference between students.

We have already discussed where there is a mismatch between the data and the encoding while simultaneously having a match between the encoding and the analysis method, so we will not repeat our comments here. Instead we now turn to the second paper where the use of a different encoding and a small difference in the analysis methodology allows for a match between data, encoding, and analysis method.

In recent work by the current authors, we used cluster analysis to group students by their responses to the FMCE [58]. This focus on the actual responses which students gave to each question allowed us to choose an encoding method that was "blind" to the various models (including the correct one). Compare that to all of the above analysis methods, which needed knowledge of the various models in order to encode the data. Our encoding recorded the letter of the response that the students chose for each question, with one exception: the response "J. None is correct" was coded as 0 because, as we discussed in Sec. III A 1, this is an opt-out response, which does not provide any definitive information about the answer the students actually want to give. We were following our own advice from earlier in this paper and picking an encoding that was well matched to the sort of data provided by the questions with which we were dealing (asymmetric nominal).

Answers encoded as letters, however, are obviously not well suited to the Euclidian distance, which Ding and Beichner used. Instead we made use of a distance measure derived from the Jaccard coefficient [59]. This distance was, therefore, well matched to our encoding, giving us a high degree of consistency from data source, to encoding, to analysis.

Thus far, the examples of analysis methods that we have examined have made use of some form of multiple-choice instrument in order to gather their data. We have done this because the use of multiple-choice surveys is so prevalent in PER and they lend themselves easily to quantitative analysis methods. The match between data source, encoding, and analysis method is, however, an issue that needs to be considered with all data sources. To illustrate this, we consider a third example of cluster analysis in which the data source was not a multiple-choice instrument.

In earlier work by the current authors, we wanted to use cluster analysis to sort student responses into groups based on similarity of responses to a short answer question [60]. Students were shown the trajectory of a moving object and given a written description of the motion the object underwent as it followed that trajectory. Along that trajectory, four points were marked off, and the students were asked to draw arrows in the direction of the acceleration of the object at those points. These questions were based on similar ones asked at the University of Washington, which have been used to reveal student difficulties with motion in two dimensions [61].

In order to define how similar (or different) two responses were, we developed a coding scheme in which each arrow that the students drew was described by a series of features which the arrow might exhibit. We identified 12 features in the data set. Two of the arrows that students drew were then examined for each feature and rated as either having or not having that feature [62]. The code for a particular student's answer, then, consisted of a series of 24 binary (nominal) variables (12 for each arrow, with the order of the features being fixed for all students). However, because the absence of a particular feature does not describe what the students are doing, most of these variables indicate the absence of that particular feature. Indeed, we noted in the original paper that each student had, on average, $2.51 \pm 0.83$ of the features [60].

We used this descriptive encoding scheme to reduce the amount of interpretative bias in the process. It was, generally, very clear whether or not a given arrow exhibited each of the features we were looking for, and in that sense the encoding scheme was well matched to the data. However, the list of features we decided were important (and thus coded for) and the list of those we decided were not (and thus did not code for) were informed by our previous experience with the question (and prior research on similar questions). This likely created biases in the encoding that prevent it from being considered a very good match. Additionally, students never saw the list of features (it was developed as we reviewed their answers) and so it would be disingenuous to say that students whose arrows did not have certain features were actively rejecting those possibilities. This is something that is easily overlooked in 
this encoding, and it led to a significant error in our original work.

In order to quantify the difference between the responses that two students gave, we originally selected the squared Euclidian distance. This required that the values of "present" and "absent" for each feature be assigned a numerical value so that the difference between two students on a particular feature $\left(A_{i}-B_{i}\right)$ could be calculated. While this may seem to be a straightforward procedure, e.g., simply assign a value of 1 to present and 0 to absent, that straightforwardness hides an assumption that must be made about the data. Since $A_{i}-B_{i}=0$ whenever $A_{i}=B_{i}$, the values of present and absent are given equal weight in measuring the difference between two students. However, since the vast majority of the features were absent for any given student, finding that two students both lacked a particular feature (and thus had absent values on that feature) did not actually provide any information about how those two students compared to each other. In other words, the values of present and absent were asymmetric in the value of their information. Since the squared Euclidian distance treats them as symmetric, it is an inappropriate distance to be using for this kind of data. Our analysis method, in other words, was not well matched to our encoding. An appropriate distance, one which is better matched to the encoding, is one that takes into account this asymmetry.

As we pointed out in our Erratum [63], one distance that does take into account this asymmetry is the Jaccard distance. Fortunately for our work, switching to the Jaccard distance did not change the conclusion that cluster analysis could be used to sort student responses. It did, however, change the organization and structure of the cluster analysis solution that we used to derive our groups. This means that if our work had gone beyond showing that cluster analysis could work, and had drawn conclusions based on the results of the cluster analysis, then those results could have been modified or invalidated by switching to the Jaccard distance.

While we still believe that cluster analysis does show promise in being able to sort student responses [63], the above discussion should make clear that it is easy to overlook how well the encoding scheme matches up to the analysis method, even for authors who are aware of the issue. It was only when we were more explicit about our data model that we were able to recognize and correct the mismatch between encoding and data analysis.

\section{E. Epistemological agreement}

The final analysis method to be considered here is the one associated with the MPEX. The MPEX, developed by Redish et al. [28], is designed to assess "student attitudes, beliefs, and assumptions about physics." It presents students with a series of statements about physics. These statements deal with multiple aspects of a student's ideas about learning physics (with categories labeled independence, coherence, concepts, reality link, math link, and effort) and are presented in both positive form (i.e., agreeing with the statement would be considered favorable by an expert) and negative form (i.e., agreeing with the statement would be considered unfavorable by an expert). For each of these statements, students are asked to rank their agreement with the statement on a 5-point Likert scale: Strongly Disagree (1), Disagree (2), Neutral (3), Agree (4), and Strongly Agree (5).

We note that Redish et al. did not do what is often done with Likert-scale data, namely, take an average of the class's score. As discussed above, ordinal data are not interval data. In this case, the difference between neutral and agreeing might be far more important than the difference between agreeing and strongly agreeing. The former (a difference from 3 to 4 , i.e., 1 unit) and the latter (a difference 4 to 5 , again 1 unit) are mathematically identical, but the intervals are possibly quite different. Thus, averages are inappropriate in this case.

Instead, student responses were compared to a set of expert responses. These were determined by administering the MPEX to "college and university teachers implementing Workshop Physics in their classroom." The majority response for each item was classified as the "expert response." [64]. A student giving this same response was said to give the favorable response, while one giving the opposite was said to give the unfavorable response.

To encode data from the MPEX to look at favorable and unfavorable scores, researchers first subtracted 3 from scores of $1-5$, such that neutral answers were scored a 0 , disagreeing was negative $(-1$ or -2$)$, and agreeing was positive (1 or 2). Then, using a question-by-question multiplication factor of either -1 or +1 , responses were converted to positive when favorable and negative when unfavorable. In this fashion, the distinction between strongly disagree and disagree, or strongly agree and agree, was removed from the data. All positive values were favorable, and all negative values were unfavorable. The encoding process had turned ordinal data from the Likert-scale questions into nominal data, defined by three categories which described all possible outcomes: favorable, neutral, and unfavorable. To analyze these data, then, researchers could count the number of positive, zero, and negative values in the processed data and see what percent of a class was answering favorably, neutrally, or unfavorably, respectively.

We believe that through these actions, Redish et al. attempted to address the previously discussed issues with Likert-scale questions regarding within- and betweenstudent comparisons. By encoding data on the MPEX in a way that allowed for analysis of the class, rather than the individual student, their approach was much like in model analysis, described above. As with model analysis, then, the interpretation of a favorable or unfavorable plot might be to describe the probability that a student in the class had favorable or unfavorable expectations. 
Analysis of the MPEX is different from model analysis in several ways, though. Model analysis does not rely on ordinal data. By removing ordinal information from the MPEX, researchers created symmetric nominal data, losing information about their students. On a student-by-student basis, we might observe the consequences of such a choice. If we imagine two students where one gives answers identical to experts and the other gives answers that are similar but chosen less "strongly," their answers would be encoded identically and they would have the same favorable or unfavorable score (both would get a 100 or 0). However, there would clearly be a difference between the two students, and one might reasonably identify one who is more closely aligned with the expert's choices than the other. Such information is lost in the method described above.

The calculation of scores, however, does not lead to an additional loss of information the way it did above for the normalized gain. Much of the information lost in computing the favorable score is retained when computing the unfavorable score and vice versa. Furthermore, the percentage of neutral (or blank) answers is simply the balance of these two scores. Since the analysis concerns percentages and three possible states, once two scores are known, no additional information is gained by calculating the third. As a result, the use of paired favorable and unfavorable scores is consistent with the nature of the encoded data and the goal of describing a class instead of individual students, because the data were encoded in a way that turned ordinal data into nominal data.

A separate issue with the analysis of the MPEX arises from the ways that missing data and neutral data are confounded. Students who do not answer a question are not necessarily neutral, maybe not even close to neutral. They could have a very strong opinion on the subject and would likely pick 1 or 5 , but may not have gotten to that question (or left it blank for any number of other reasons). Not answering a question should not, therefore, be treated the same as choosing neutral. This distinction is lost in the encoding used by Redish et al.

If desired, one could address the loss of information in the encoding process by keeping the ordinal information and by not combining the strongly (dis)agree and (dis)agree responses in the two categories of favorable and unfavorable responses. At that point, issues of comparing ordinal data would arise. First, we would need to decide whether we are going to interpret the data as within-students ordinal data or between-students ordinal data. As we indicated in Sec. III A 4, Likert-scale questions can be interpreted from two different points of view: one in which each student ranks the items against each other (leading to withinstudents ordinal data) and in which each item ranks the students against each other (leading to between-students ordinal data). This choice is not trivial, as it makes a significant difference as to the type of research questions we can answer using the data. While we have thus far avoided discussing how the research question can affect the encoding decisions that are made, we will explore it here in order to provide the reader with an example of how those interactions might play out.

Using the point of view which leads to within-students ordinal data, we will be able to answer questions that deal with the overall epistemological understanding of a single student and how those students compare to each other. We could also compare how a student compares to some predefined "ideal" ranking (such as the "expert's choice" responses). With a suitably defined centroid-finding function, the "typical" response of a group of students could be found, allowing comparisons of various classes, experimental groups, demographic groups etc., to each other or to the "ideal" response.

Using the point of view that leads to between-students ordinal data, we will instead be able to answer questions geared towards granular aspects of the epistemological view of a group of students. That is, how do the various statements compare to each other in the way they rank the students? Which items go together in the sense that they tend to rank the students the same way? Are all the statements within a particular dimension of student expectations coherent, in that they all tend to rank the students in the same way? How does the ranking of the students by the item compare to some external student ranking, such as that derived from the students grades? Again, with a suitably defined centroidfinding function we can move from answering these questions for particular items to answering them for groups of items.

The questions Redish et al. [28] were most interested in seemed to fall primarily into the first group, so it would make sense to choose the within-students point of view. Before settling on an encoding, though, we would want to look at our analysis tools so that we can have a good match at both ends of the encoding bridge.

For example, let us assume that we want to compare students to each other (either individually or as groups) by how they ranked the items on the MPEX. In Sec. III B, we described two measures for computing the similarity between ordinal data sets: the Spearman rank correlation and Kendall's $\tau$. The first, as we pointed out, makes use of the mean rank and the standard deviation of the ranks. If we were to use the Spearman rank correlation, we would want an encoding scheme to make mean rank and the standard deviation of the ranks be either constant (and so not affect the analysis) or meaningful (and so represent something about the data). In this instance, we suggest that the ranks run from 1 to 34, and the mid-rank method for handling ties be used. Kendall's $\tau$, on the other hand, only looks at the relative order of ranking; how many ranks there are overall and in between any two items does not matter when performing this calculation. Our encoding scheme would simply use the numerical responses 1 to 5 . Since this is a much easier encoding process, it is the one which we would recommend. That said, one should only carry out the more difficult process if it aligns with one's research goals and helps answer one's questions. 
On the other hand, for a detailed validity study of a test like the MPEX, the between-students point of view would be more appropriate. In this case we would be primarily interested in comparing the items to each other to ensure that those meant to be getting at the same thing are well correlated and those which are meant to be independent are not (at least relatively speaking). As with the withinstudents point of view, the analysis method we intend to use for comparing the items to each other will affect our encoding scheme, and so careful consideration is necessary before committing to a particular encoding scheme.

Finally, we should point out that these decisions are not necessarily mutually exclusive. It is possible to start with the between-students point of view, for instance, and then ask questions about the relative positions of particular students (or groups of students) within the ranking scheme of a particular item (or group of items). However, the way one goes about conducting the analysis in order to answer those questions will change depending on the point of view that one started from. As a result, there should be some very careful and explicit consideration of this decision when reporting on research of this kind.

\section{CONCLUSIONS}

In this paper, we have discussed the role that data encoding can play in moving from the raw information that one gathers from written or survey data to the analysis that helps researchers make claims that answer their research questions. To do so, we started with a theoretical discussion of the nature of data, which focused on the mathematical operations that are allowed to be carried out with the various types of data. This allowed us to consider how much information is kept when encoding and what kinds of analysis are possible with that particular kind of data. We then looked at the issues of encoding and analysis in PER separately. To discuss encoding, we used examples from the PER literature to define the data gathered by the questions typically used in our field (e.g., free response, multiple choice, Likert scale, ranking task, grades, etc.). This allowed us to discuss how these data can be encoded to preserve information. We then used the example of distances or correlations to show how different encodings match up to different forms of analysis. Finally, to discuss analysis more generally, we looked at examples from the literature to describe different ways in which encoding and analysis were, or were not, consistent.

Throughout, we have highlighted possible pitfalls in the encoding process. These pitfalls affect the conclusions that can be made during the analysis. For example, one might misinterpret the nature of the data while encoding and use an inappropriate distance measure when carrying out cluster analysis, as in our own work [60]. Or, as is more common, one might try to take averages of data for which the concept of average is meaningless.

A consistent area in which we observe the effects of data encoding are where one loses information in the data during the encoding process. This decision often has profound effects on the analysis process. We discussed the example of Redish et al.'s study on student expectations using the MPEX, where ordinal data was converted into nominal data, which contains less information. This conversion allowed the authors to carry out an analysis that emphasized the overall expectations of a group rather than the specific expectations of a single student. The analysis was thus consistent with the encoding.

We also discussed examples where information about well-formed distractors on multiple-choice questions is dropped during the encoding process. For example, when using normalized gain, one only focuses on the right answers and ignores information that is in the incorrect answers, even though tests like the FCI and FMCE were created with clusters of common wrong answers in mind. Similarly, in many of the factor analyses carried out on either the FCI or FMCE, the focus on only right and wrong choices limits the ability to analyze what students might be thinking. Encoding data with the goal of using a different correlation coefficient (e.g., simple matching, Pearson, Spellman, or Kendall's $\tau$ ) which is better matched to the properties of the data may remove some of these limits.

We have also highlighted examples where possible problems with data encoding were addressed and the encoding process was closely aligned with the analysis. In model analysis, in contrast to normalized gain, the information that is contained in the incorrect multiplechoice answers is used to determine the probability of answering questions using alternative models. Though originally described for situations in which there is a community consensus and a single most common incorrect model [56], model analysis has been extended to situations where multiple common models are used by students in a single situation [57]. In a second example, we used our own work [63] to show that more careful attention to the nature of the data being encoded allowed us to choose a more appropriate distance measure in a cluster analysis.

Our examples show that the process of encoding data plays an essential role in the subsequent analysis. However, we find that authors typically provide little information about how their data were encoded. Often, the process has to be gleaned from the details of the analysis, and the role of assumptions made by the authors is often unclear. More powerfully, mismatches between the data and its encoding or the encoding and the analysis may lead to inappropriate conclusions and claims that are not actually backed up by the data.

In sum, our article is meant as an introduction to a topic that receives little attention in the PER literature and as a call for further discussion and clarity in our work, so that our claims are better justified and our work has greater impact in the broader science education community.

\section{ACKNOWLEDGMENTS}

The work described in this paper was supported in part by NSF Grant No. REC-0633951. 
[1] L. Louca and D. Hammer, Student nascent abilities for scientific argumentation: The case of a 5th-6th-grade conversation about a dropped pendulum (to be published).

[2] M. R. Anderberg, Cluster Analysis for Applications, edited by Z. W. Birnbaum and E. Lukacs, Probability and Mathematical Statistics: A Series of Monographs and Textbooks (Academic Press, New York, 1973), Vol. 19.

[3] L. Kaufman and P. J. Rousseeuw, Finding Groups in Data: An Introduction to Cluster Analysis, Wiley Series in Probability and Mathematical Statistics: Applied Probability and Statistics (Wiley, New York, 1990).

[4] P.-N. Tan, M. Steinbach, and V. Kumar, Introduction to Data Mining (Pearson Education Inc., Upper Saddle River, NJ, 2006).

[5] A. K. Jain and R. C. Dubes, Algorithms for Clustering Data, Prentice Hall Advanced Reference Series (Prentice Hall, Englewood Cliffs, NJ, 1988).

[6] R. C. Dubes and A. K. Jain, Clustering methodologies in exploratory data analysis, in Advances in Computers, edited by M. C. Yovits (Academic Press, New York, 1980), Vol. 19, pp. 113-228.

[7] It is possible to make an argument for different treatment on the basis of practicality. Operators that are appropriate to discrete data (e.g., Kronecker delta $\delta_{i j}$ ) can be replicated using other mathematical tools [e.g., the squared difference $\left.(i-j)^{2}\right]$ if the encoding is right (e.g., states of 1 and 0$)$. This allows one to use tools designed for other kinds of data with binary data while still respecting the discrete nature of the data. However, this tends to blur the lines between the various range sets and should thus be undertaken explicitly after due consideration and will not be considered in this theoretical discussion.

[8] Some mathematicians would add exponentiation $\left[x^{y}\right.$ and $\left.\log _{x}(y)\right]$ to this list of distinct mathematical operations, e.g., Refs. $[9,10]$. but we have not encountered any literature on data analysis which does so, and so will ignore it.

[9] K. Devlin, It ain't no repeated addition (2008), https:// www.maa.org/external_archive/devlin/devlin_06_08.html.

[10] K. Devlin, It's still not repeated addition (2008), https:// www.maa.org/external_archive/devlin/devlin_0708_08 .html.

[11] Since some mathematicians insist that multiplication and exponentiation are basic operations, distinct from addition, e.g., Refs. $[9,10,12,13]$, an argument could be made that there exist types of data for which addition, multiplication, or exponentiation is defined without the other two processes being defined (interval being the one with addition but not multiplication or exponentiation), other, higher types for which two of these processes but not the third are defined (ratio being the one with addition and multiplication but not exponentiation), and a final, top type for which all three are defined. However, we know of no literature on data analysis that makes said argument, nor do we see a reason for advancing such a system as better than the one usually proposed.

[12] K. Devlin, Multiplication and those pesky British spellings (2008), https://www.maa.org/external_archive/devlin/ devlin_09_08.html.
[13] K. Devlin, Repeated addition — one more spin (2010), https:// www.maa.org/external_archive/devlin/devlin_01_10.html.

[14] In most of the literature on data with which we are familiar, this possibility is only treated with respect to binary data, e.g., Refs. [2,3]. However, as was noted in Tan et al. [4] binary data are a special case of nominal data, and thus this asymmetry can be generalized to nominal data relatively easily. In PER, the FMCE uses "None of the above" on nonbinary data, as well.

[15] The reader should look at Sec. 1.3 in Ref. [16] for an alternative way of interpreting this difference.

[16] M. G. Kendall, Rank Correlation Methods (Charles Griffin \& Company Limited, London, 1962).

[17] We note that this is a personal recommendation from the authors based on preserving the meaning of rank differences, not something we have encountered in the literature. Indeed, we have found no mention of how to interpret rank differences and deal with ties simultaneously.

[18] If this claim is not obvious to the reader, then consider a situation in which a ten question survey has been given to ten students. This would result in 100 student or response pairs which could be arranged in a $10 \times 10$ table where each row represented a single student and each column a single question. If six of these student or response pairs were missing, however, there would be holes in this table of data. In the case where all six of the missing pairs were confined to a single student or question, then the elimination of that student or question would result in the loss of only four student or response pairs for which data was actually taken. On the other hand, if all six of the missing student or response pairs were for different students and questions, then it would require the elimination of up to six students or six questions (or some combination thereof) to eliminate all the holes. As a result between 45 (eliminating 3 questions and 3 students) and 54 (eliminating 6 students or 6 questions) student or response pairs for which data was actually taken would also be eliminated. Given that just 94 student or response pairs were taken in total, this is around half of the data set.

[19] E. F. Redish, Teaching Physics with the Physics Suite (Wiley, New York, 2003).

[20] R. K. Thornton and D. R. Sokoloff, Assessing student learning of Newton's laws: The Force and Motion Conceptual Evaluation and the Evaluation of Active Learning Laboratory and Lecture Curricula, Am. J. Phys. 66, 338 (1998).

[21] D. Hestenes, M. Wells, and G. Swackhamer, Force concept inventory, Phys. Teach. 30, 141 (1992).

[22] C. J. Hieggelke, D. P. Maloney, S. E. Kanim, and T. L. O'Kuma, E\&M TIPERs: Electricity \& Magnetism Tasks Inspired by Physics Education Research (Pearson Education Inc., Upper Saddle River, NJ, 2006).

[23] M. C. Wittmann, R. N. Steinberg, and E. F. Redish, Making sense of how students make sense of mechanical waves, Phys. Teach. 37, 15 (1999).

[24] L. C. McDermott, P. S. Shaffer, and the Physics Eductation Group, Tutorials in Introductory Physics (Prentice Hall, Englewood Cliffs, NJ, 2002).

[25] D. P. Maloney, Ranking tasks: A new type of test item, J. Coll. Sci. Teach. 16, 510 (1987). 
[26] D. P. Maloney and A. W. Friedel, Ranking tasks revisited: Teaching students to solve complex scientific problems, J. Coll. Sci. Teach. 25, 205 (1996).

[27] Ranking Task Exercises in Physics, edited by T. L. O'Kuma, D. P. Maloney, and C. J. Hieggelke (Prentice Hall, Englewood Cliffs, NJ, 2000).

[28] E. F. Redish, J. M. Saul, and R. N. Steinberg, Student expectations in introductory physics, Am. J. Phys. 66, 212 (1998).

[29] B. L. Sherin, Using latent semantic analysis and cluster analysis to automate the analysis of clinical interview data, in Annual Meeting of the American Educational Research Association (American Educational Research Association, San Diego, CA, 2009).

[30] R. P. Springuel, Applying cluster analysis to physics education research data, Ph.D. thesis, University of Maine, Orono, ME, 2010.

[31] R. R. Sokal and P. H. A. Sneath, Principles of Numerical Taxonomy, edited by D. M. Whitaker, R. Emerson, D. Kennedy, and G. W. Beadle, A Series of Books in Biology (W. H. Freeman and Company, San Francisco, CA, 1963).

[32] D. J. Rogers and T. T. Tanimoto, A computer program for classifying plants, Science, New Series, 132, 1115 (1960).

[33] P. Jaccard, Nouvelles recherches sur la distribution florale, Bulletin de la Société Vaudoise des Sciences Naturelles 44, 223 (1908).

[34] L. R. Dice, Measures of the amount of ecologic association between species, Ecology 26, 297 (1945).

[35] M. de Hoon, S. Imoto, and S. Miyano, The C clustering library, Online (2010).

[36] C. Spearman, The proof and measurement of association between two things, Am. J. Psychol. 15, 72 (1904).

[37] F. W. Gery, Is there a ceiling effect to the Test of Understanding in College Economics?, in Research Papers in Economic Education, edited by A. L. Welsh (Joint Council on Economic Education, New York, 1972), pp. 35-49.

[38] C. I. Hovland, A. A. Lumsdaine, and F. D. Sheffield, Appendix A-A baseline for measurement of percent change, in Experiments on Mass Communication, Studies in Social Psychology in World War II, Vol. III, edited by F. Osborn, L. S. Cottrell, Jr., L. C. DeVinney, C. I. Hovland, J. M. Russel, S. A. Stouffer, and D. Young, Ex Officio (Princeton University Press, Princeton, New Jersey, 1949).

[39] R. R. Hake, Interactive-engagement versus traditional methods: A six-thousand-student survey of mechanics test data for introductory physics courses, Am. J. Phys. 66, 64 (1998).

[40] T. I. Smith and M. C. Wittmann, Applying a resources framework to analysis of the Force and Motion Conceptual Evaluation, Phys. Rev. ST Phys. Educ. Res. 4, 020101 (2008).

[41] Technically, there is a single free response question on the test, 46a in the energy cluster, but this question is usually ignored when analyzing the FMCE, including in the work by Ramlo, and so will also be ignored here.

[42] Template for analyzing the FMCE (2001), http://umaine .edu/per/wp-content/uploads/sites/436/2009/12/template_ fmce.zip.

[43] D. I. Dykstra Jr., Against realist instruction: Superficial success masking catastrophic failure and an alternative, Constructivist Found. 1, 49 (2005).
[44] J. D. Marx and K. Cummings, Normalized change, Am. J. Phys. 75, 87 (2007).

[45] L. Bao, Theoretical comparisons of average normalized gain calculations, Am. J. Phys. 74, 917 (2006).

[46] Harder here is a relative term. Depending on how the data are stored, the process of going from letters to a score can be made trivial by use of the right tools. For instance, the spreadsheet analysis tool for the FMCE mentioned above has a formula in it which makes the conversion automatic as the data are entered.

[47] S. Ramlo, Validity and reliability of the force and motion conceptual evaluation, Am. J. Phys. 76, 882 (2008).

[48] D. Huffman and P. Heller, What does the force concept inventory actually measure?, Phys. Teach. 33, 138 (1995).

[49] L. Ding and R. Beichner, Approaches to data analysis of multiple-choice questions, Phys. Rev. ST Phys. Educ. Res. 5, 020103 (2009).

[50] D. Hestenes and I. A. Halloun, Interpreting the force concept inventory: A response to March 1995 critique by Huffman and Heller, Phys. Teach. 33, 502 (1995).

[51] L. Ding, Designing an energy assessment to evaluate student understanding of energy topics, Ph.D. thesis, North Carolina State University, Raleigh, North Carolina, 2007.

[52] The last response, (d), for question 1 starts as a "None of the above" response, but then adds information that makes selecting it more than just a negation of the other responses. Similarly, responses (b) and (e) for question 22 have "Not enough information" in them, but also have additional responses which make them more than just a negation of other responses.

[53] J. Carroll, The nature of the data, or how to choose a correlation coefficient, Psychometrika 26, 347 (1961).

[54] In their response to criticism by Hestenes and Halloun [50], Heller and Huffman [55] argue in favor of their correlation coefficient choice by showing that the full range of values for the correlation coefficient can be achieved in two hypothetical populations: one of which is $80 \%$ correct and the other of which is $40 \%$ correct. In both cases they seem to have assumed that all questions had the same percentage of students answering correctly. That is, they did not look at what would happen if in a single population one question was answered correctly by $80 \%$ of the students and another was answered correctly by $40 \%$ of the students.

[55] P. Heller and D. Huffman, Interpreting the force concept inventory: A reply to Hestenes and Halloun, Phys. Teach. 33, 503 (1995).

[56] L. Bao and E. F. Redish, Model analysis: Representing and assessing the dynamics of student learning, Phys. Rev. ST Phys. Educ. Res. 2, 010103 (2006).

[57] T. I. Smith, M. C. Wittmann, and T. Carter, Applying model analysis to a resource-based analysis of the Force and Motion Conceptual Evaluation, Phys. Rev. ST Phys. Educ. Res. 10, 020102 (2014).

[58] R. P. Springuel, M. C. Wittmann, and J. R. Thompson, Using cluster analysis to group student responses on the force and motion conceptual evaluation (to be published). 
[59] The Jaccard coefficient is a measure of how similar two response sets are. Since our cluster analysis methods worked by looking for differences between student responses, we had to "invert" the Jaccard coefficient to get a distance which measured how different they were.

[60] R. P. Springuel, M. C. Wittmann, and J. R. Thompson, Applying clustering to statistical analysis of student reasoning about two-dimensional kinematics, Phys. Rev. ST Phys. Educ. Res. 3, 020107 (2007).

[61] P. S. Shaffer and L. C. McDermott, A research-based approach to improving student understanding of the vector nature of kinematical concepts, Am. J. Phys. 73, 921 (2005).
[62] We did not use all 4 arrows in order to simplify the data set. This study was intended as a proof of concept to show that cluster analysis could be used to do the task, not as a full examination of the data.

[63] R. P. Springuel, M. C. Wittmann, and J. R. Thompson, Erratum: Applying clustering to statistical analysis of student reasoning about two-dimensional kinematics [Phys Rev. ST Phys. Educ. Res. 3, 020107 (2007)], Phys. Rev. ST Phys. Educ. Res. 5, 029902(E) (2009).

[64] For all but three items this majority was around $80 \%$. For those remaining three it was between $66 \%$ and $75 \%$. 